INTERNATIONAL JOURNAL OF MULTIDISCIPLINARY RESEARCH AND ANALYSis

ISSN(print): 2643-9840, ISSN(online): 2643-9875

Volume 05 Issue 01 January 2022

DOI: 10.47191/ijmra/v5-i1-25, Impact Factor: 6.072

Page No.- 205-210

\title{
The State of the Nasal Cavity of Patients with Exudative Otitis Media
}

\author{
U.S. Khasanov' ${ }^{1}$, G.A. Rakhimjonova ${ }^{2}$, G.S. Khaydarova ${ }^{3}$, J.A. Djuraev ${ }^{4}$ \\ 1,2,3,4 Tashkent Medical Academy
}

\begin{abstract}
The state of intranasal structures was compared in patients with EOM and AR and EOM without AR. It was determined that the most likely cause of the violation of the protective function of the nasal mucosa in patients with EOM and AR is serous inflammation, manifested by a number of structural and functional changes. Structural changes are characterized by deepithelialization of the mucous membrane in the acute phase, an increase in the number of goblet cells, a change in the viscosity of the medium, and a change in the $\mathrm{pH}$ of the medium. Each of the described processes ultimately leads to a slowdown in mucociliary transport, which occurs in the patients with EOM and AR that we examined. Thus, the curvature of the nasal septum and other features of the architectonics of intranasal structures in patients with EOM to a lesser extent than in patients with EOM and $A R$ affect the development of the disease.
\end{abstract}

KEYWORDS: Exudative Otitis Media, Allergic Rhinitis, Nasal Cavities, Ciliary Epithelium, Deviation Of Septum Of The Nose.

\section{INTRODUCTION}

The prevalence of exudative otitis media (EOM) is especially high among children: according to some foreign authors, the disease occurs in $5-25 \%[1,2,6]$, and others - in 6.5 to $10.9 \%$ [4]. This indicator reaches its maximum values at the age of 2-5 years, and by the age of 10, 80 children have a history of at least one episode of EOM [3]. As noted by B. Sedlmeier et al. (2009) [7], boys are more susceptible to the disease. In studies, a number of authors have shown [5] that in Europe EOM is the main cause of hearing loss in children aged 2 to 7 years (during mass examinations of children in this age group, it is found in 30.2 cases) [11]. The high frequency of EOM in children - 4 times more often than in adults - is associated with anatomical prerequisites: a short horizontal auditory tube contributes to infection of the middle ear cavity $[8,9,10]$.

The development of exudative otitis media can be promoted by inflammatory diseases of the nose and paranasal sinuses, hypertrophy of the lymphoid tissue of the pharynx, cicatricial changes in the nasopharynx, paresis of the muscles of the soft palate, deviated nasal septum, as well as malignant neoplasms of the nasopharynx [6], if they interfere with the drainage function of the auditory tube. However, the presence of these diseases in itself is not always accompanied by the development of EOM [12,13].

The results of O.V. Stratieva (1999) showed the importance of the architectonics of the middle ear cavity and pneumatization of the mastoid process in the development of EOM. The high dome of the jugular vein in the hypotympanum in combination with closely spaced ridges in 65 cases contributes to the formation of cysts, early fibrosis, and the low topic of the vein in 45 cases predisposes to prolonged exudation in the area between the hyporethrotympanum and latent mucoperiostitis in the zone of the posterior paralabyrinth and parafacial cells [14].

In the works of K. Apostolopulos et.al. [15] found a relationship between blood type and predisposition to EOM: in representatives of II (A) and III (B) blood groups, the incidence of otitis was higher than in people with I (O) and IV (AB) blood groups. Hereditary genetic predisposition also matters: according to S.N. Loginova (2005) [14], latent dysfunction of the auditory tube was found in 98 Evenki schoolchildren, which explains their high incidence of otitis media (360.8\%). In an experimental study by F.F.Depreux et.al. [16] found that the absence of the Eya4 transcription activator in mice leads to abnormal structuring of the Eustachian tube, being a predisposing factor in the development of EOM. In patients with congenital cleft lip and palate, there is a pathological attachment of the muscles that stretch and lift the palatine curtain to the auditory tube, which causes a high prevalence (up to 56) of exudative otitis media in this group of patients [4]. 


\section{The State of the Nasal Cavity of Patients with Exudative Otitis Media}

A relationship has been found between certain perinatal factors (caesarean section, preterm birth) and a tendency to recurrent EOM, which the authors attribute to a deficiency of the surfactant system in the eustachian tube epithelium [2]. In the study by E.V. Garova et al. EOM is considered as an early manifestation of Wegener's granulomatosis [6].

J.L. Desseyn suggested the role of modified mucin as a result of genetic disorders, which may be the reason for the increase in mucus viscosity [17].

M.Yu. Boboshko and T.V. Klimantseva in their studies pay attention to the presence of a vegetative imbalance with a predominance of the influences of the parasympathetic nervous system as one of the factors predisposing to the occurrence of exudative otitis media. $[12,18]$

O.T. Petukhova suggested the role of endothelial dysfunction in the violation of vascular permeability and, as a result, the occurrence of tubal disorders [19].

In the study [6] of exudate from the middle ear in patients with EOM for the presence of free radicals in all samples, an increased content of lipid peroxidation products, known for their altering effect, was found.

Also, in the works of domestic and foreign authors, it is indicated that pharyngolaryngeal reflux can become one of the causes of the development of exudative otitis media - with concomitant pathology of the gastrointestinal tract, reflux was detected in 83.7 patients with EOM [14].

Given the sharp increase in allergic diseases over the past decades, the authors began to study the role of allergic, immune and autoimmune mechanisms in the development of exudative otitis media [8]. So, when observing a large group of children suffering from bronchial asthma or allergic rhinitis (AR), it was found that 30 children had otoscopic changes in the middle ear, while $5 \%$ of them had a type B tympanogram, and 20 had a type B tympanogram. At the same time, according to some authors, it is difficult to say with certainty whether exudative otitis media is an allergic disease or just the result of allergic edema of the nasopharyngeal mucosa [20]. Other researchers believe that it is an unfavorable allergic background, concomitant allergic diseases or a local allergic reaction that play a leading role in the occurrence of exudative otitis media [21].

\section{MATERIAL AND METHODS}

From 2017 to 2021, we conducted a comprehensive examination and treatment of 220 children who were at the pediatric ENT department of the MDC of TMA with exudative otitis media, who underwent clinical, audiological, allergeological, immunological and laboratory-instrumental studies on the effectiveness of complex treatment. in children of the younger age group, there is an insufficient detection of this disease. So, for example, the parents of 63 children, who had adenoids and acute rhinosinusitis, did not go to the doctor earlier with complaints of hearing loss. Prior to admission to the clinic, conductive hearing loss was detected in 134 children. Previously, 102 children received treatment for EOM. EOM is twice as common in boys as in girls. Seasonal changes in the incidence of EOM are also noticeable, which increase in the autumn-winter period.

Among the patients we observed, there were 65 children (unilateral processes), and 135 children (bilateral processes) suffering from acute EOM.

Acute EOM identified:

- In the primary phase - in 53 children $(29,4 \%)$

- In the secretory phase - in 123 children $(68,3 \%)$

- In the fibrinous phase - in 24 children $(22,4 \%)$

\section{One of the reasons that preceded the development of the EOM were:}

- Adenoids and rhinosinusitis - in $43 \%$ of children;

- atrophic and cicatricial changes in the area of the pharyngeal mouth of the auditory tube - in $5 \%$ of children;

- local malformations of the nasal cavity - in $15 \%$ of children;

- vasomotor salpingo-otitis and rhinitis - in $8 \%$ of children;

- $\quad$ hypertrophy of tubal folds - in $8 \%$ of children;

- $\quad$ adhesions of the auditory tube - in $3 \%$ of the studied children.

- physiological obstruction of the pharyngeal mouth of the auditory tube in $18 \%$ of children;

- adhesions of the auditory tube - in $3 \%$ of the studied children

We have noted a change in the causes of EOM, depending on the age of children. In children in the younger age group (from 3 to 6 years), adenoiditis was more common (in the amount of $43 \%$ of the studied patients), physiological obstruction of the pharyngeal mouth of the auditory tube (18\%). In children aged 7 to 11 years, local malformations of the nasal cavity (15\%), 


\section{The State of the Nasal Cavity of Patients with Exudative Otitis Media}

hypertrophy of the tubal ridges (8\%), cicatricial processes in the nasopharynx (5\%) were more common. At the age of 12 to 14 years - acute rhinosinusitis (41\%).

Patients with addiction etiology (allergic rhinitis) were divided into 2 groups:

Group 1 - patients with exudative otitis media and allergic rhinitis;

Group 2 - patients with exudative otitis without allergic rhinitis.

The control group consisted of practically healthy children aged 2 to 14 years $(6.8 \pm 1.6)$.

\section{RESULTS AND DISCUSSION}

Rhinoscopy revealed certain features characteristic of each group of examined patients. The most pronounced changes were noted in patients with EOM and AR: swelling of the nasal mucosa of varying degrees, pallor or cyanosis of it, mucous discharge in the lumen of the nasal passages. Such changes alone or in combination were detected in 107 (89.2\%) patients of the main group (EOM and AR).

In contrast, patients of group 2 (EOM) more often had hyperemia and slight swelling of the nasal mucosa, sometimes mucous discharge in the nasal passages (Figure 3).

A more detailed examination of the nasal cavity and nasopharynx was performed using a rigid endoscope (KARL STORZ). For this study, it was important to compare the rhinoscopy picture in EOM and AR and EOM. The identified changes and their frequency are shown in Table 1.

From the data presented, it can be seen that patients with EOM and AR more often had swelling of the nasal and nasopharyngeal mucosa. At the same time, the rhinoscopic picture in EOM patients did not have a clear change (Fig. 1,2,3,4).

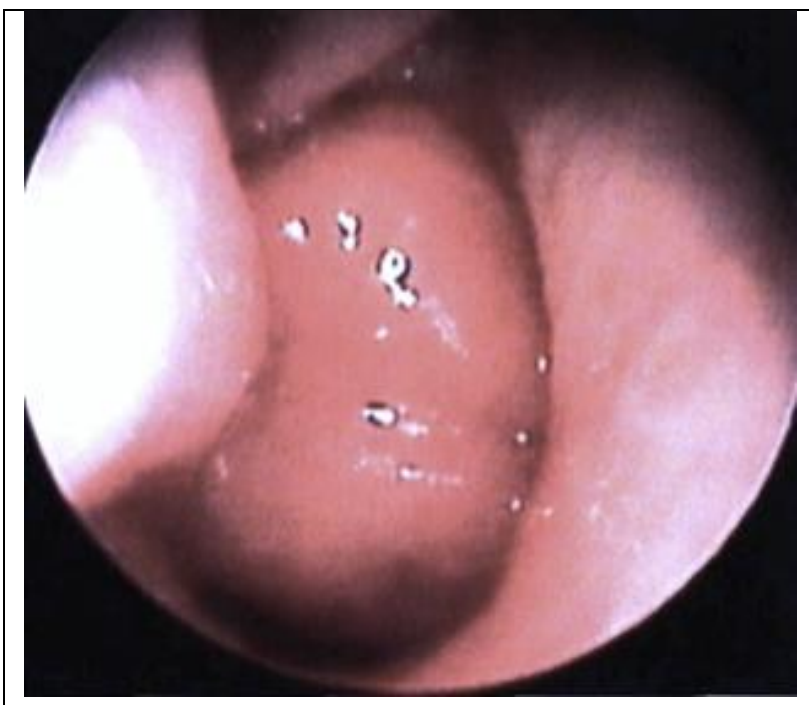

Fig.1. Adenoids of the IV degree, blocking the mouth of the auditory tube.

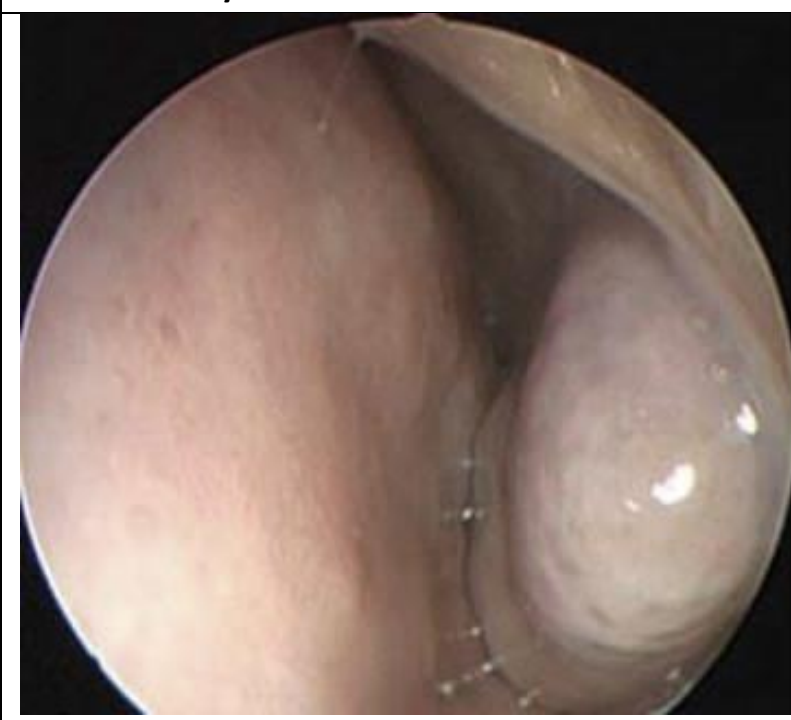

Fig. 3. Rhinoendoscopy of allergic rhinitis.

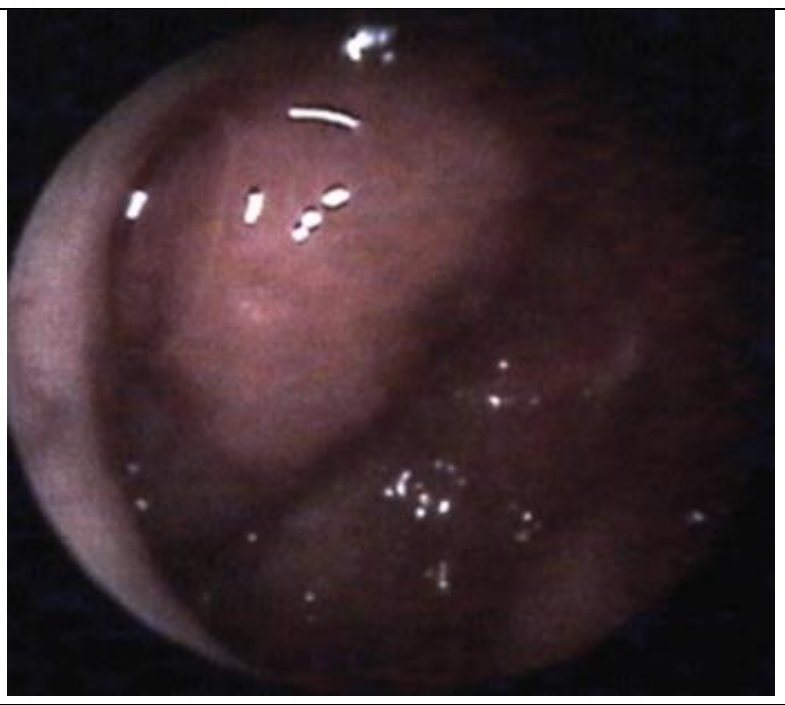

Fig. 2. Hypertrophy of the tubal tonsil in a child with the secretory stage of EOM

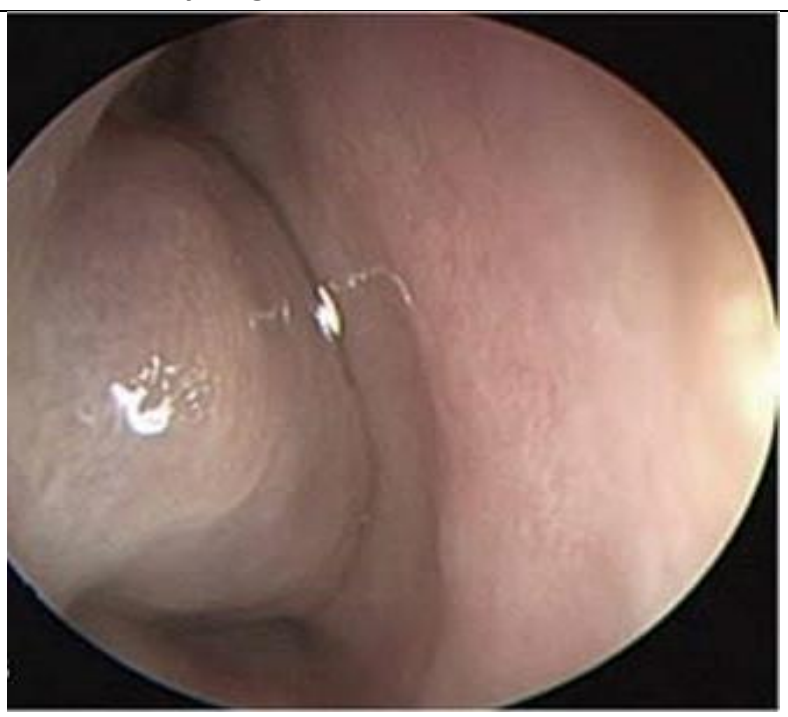

Fig. 4. Rhinoendoscopy of allergic rhinitis. 


\section{The State of the Nasal Cavity of Patients with Exudative Otitis Media}

Table 1. Rhinoscopic picture in patients of groups 1 and 2

\begin{tabular}{|l|l|l|}
\hline Identified changes (signs): & Feature frequency in the group: \% \\
\cline { 2 - 3 } & $\begin{array}{l}1 \text { group } \\
(\mathrm{n}=120)\end{array}$ & $\begin{array}{l}2 \text { group } \\
(\mathrm{n}=100)\end{array}$ \\
\hline Swelling of the nasal mucosa & 81,6 & 31,2 \\
\hline Discoloration of the nasal mucosa & 76,6 & 46,8 \\
\hline Deviation of septum of the nose & 53,4 & 56,5 \\
\hline $\begin{array}{l}\text { Edema of the mucous membrane of the } \\
\text { nasopharynx in the area of the mouths of } \\
\text { the auditory tubes }\end{array}$ & 49,6 & 30,2 \\
\hline Adenoid vegetations in the nasopharynx & 13,5 & 9,3 \\
\hline $\begin{array}{l}\text { Other anatomical features (conchabullosis, } \\
\text { large uncinate process, hypertrophy of the } \\
\text { ethmoid bulla, etc.) }\end{array}$ & 4,1 & 2 \\
\hline
\end{tabular}

In addition, Table 1 shows that deviated septum in patients with EOM and AR and EOM without AR occurred with almost equal frequency.

However, by comparing otoscopic and rhinoscopic data in patients with unilateral otitis media, we found a significant difference in the results obtained between the main group (EOM and AR) and group 2 (EOM). The results are reflected in the table 2.

Table 2. Combination of unilateral otitis media with deviated septum in patients with exudative otitis media

\begin{tabular}{|l|l|l|}
\hline $\begin{array}{l}\text { Comparison criteria: rhinoscopy data and their } \\
\text { combination with otoscopic data: }\end{array}$ & $\begin{array}{l}\text { Number of patients with unilateral otitis } \\
\text { matching criteria: \% }\end{array}$ \\
\cline { 2 - 3 } & $\begin{array}{l}1 \text { group } \\
(\mathrm{n}=120)\end{array}$ & $\begin{array}{l}2 \text { group } \\
(\mathrm{n}=100)\end{array}$ \\
\hline Deviation of septum of the nose & 57,1 & 45 \\
\hline $\begin{array}{l}\text { The direction of the curvature of the nasal septum } \\
\text { coincides with the side of the otitis }\end{array}$ & 33,6 & 26 \\
\hline $\begin{array}{l}\text { The direction of the curvature of the nasal septum } \\
\text { does not coincide with the side of the otitis }\end{array}$ & 37,2 & 32 \\
\hline
\end{tabular}

The table shows that in patients with EOM, nasal septal curvature was significantly more often $(p=0.009)$, and the direction of the curvature coincided with the otitis side significantly more often $(p=0.004)$. In the group with unilateral EOM, the difference between the number of patients with and without deviated nasal septum was insignificant $(p=0.821)$. While in the corresponding category of patients with EOM, the difference turned out to be significant $(p<0.001)$ : the ratio of the number of patients with and without deviated nasal septum was 6:1.

To clarify the degree of influence on the development of EOM and AR, and EOM of the features of the architectonics of intranasal structures, we conducted a comparative analysis of the anamnesis and rhinoscopy data (Table 3 ).

Table 3. Comparative analysis of anamnesis and rhinoscopy data in patients of both groups

\begin{tabular}{|l|l|l|}
\hline $\begin{array}{l}\text { Comparison criteria: anamnestic data and } \\
\text { their combination with rhinoscopy: }\end{array}$ & \begin{tabular}{l} 
Number of patients matching the criterion: \% \\
\cline { 2 - 3 }$(\mathrm{n}=120)$
\end{tabular} & $\begin{array}{l}\text { 2 group } \\
(\mathrm{n}=100)\end{array}$ \\
\hline history of otitis media & 60,1 & 42 \\
\hline History of repeated otitis media & 53,7 & 37 \\
\hline History of repeated unilateral otitis media & 28,6 & 16 \\
\hline $\begin{array}{l}\text { Repeated unilateral otitis media in history } \\
\text { with appropriate architectonics of intranasal } \\
\text { structures (coincidence of the direction of } \\
\text { curvature of the nasal septum) }\end{array}$ & 35,2 & 14 \\
\hline
\end{tabular}




\section{The State of the Nasal Cavity of Patients with Exudative Otitis Media}

Thus, the curvature of the nasal septum and other features of the architectonics of the intranasal structures in patients with EOM to a lesser extent than in patients with EOM and AR affect the development of the disease.

In addition, the results of a comparative analysis of the history and rhinoscopy data clearly demonstrate the dependence of relapses of exudative otitis media on the state of intranasal structures in patients of group 1 and the absence of such in patients of group 2. Therefore, we can assume that pathological changes in the intranasal structures are one of the main causes of the development of EOM. And we regard this factor only as an accompanying one. In further studies, it is required to determine the cause of the pronounced swelling of the nasal mucosa, identified in the majority of patients with EOM, and to assess its significance in the development of the disease.

It turned out that the proportion of patients who had exudative otitis media and a history of AR was only significantly higher than in other groups. However, patients with EOM were significantly more likely to have a history of recurrences of unilateral otitis media $(p=0.001)$, especially with existing pathological changes in the intranasal structures $(p<0.001)$. At the same time, the similarity of the results of the study in patients with EOM and AR is noticeable.

The study of the functions of the nose revealed a difference in changes in patients between the main and 2 groups (Table 4).

Table 4. The results of the study of the functions of the nose of patients of groups 1 and 2

\begin{tabular}{|c|c|c|c|}
\hline \multirow{2}{*}{ Indicator } & \multicolumn{2}{|c|}{ Patients with EOM ( $n=220)$} & \multirow[b]{2}{*}{ Control, $n=20$} \\
\hline & $\begin{array}{l}\text { EOM and } A R, \\
n=120\end{array}$ & $\begin{array}{l}\text { EOM, } \\
n=100\end{array}$ & \\
\hline Mucociliary clearance (min) & $31,2 \pm 2,21 * * *$ & $28,14 \pm 1,46^{*}$ & $11,8 \pm 1,4$ \\
\hline $\begin{array}{l}\text { Indicators of the concentration of } \\
\text { hydrogen ions }(\mathrm{pH})\end{array}$ & $7,42 \pm 0,06^{* *}$ & $7,1 \pm 0,03 *$ & $7,0 \pm 0,01$ \\
\hline $\begin{array}{l}\text { Suction function (pupil reaction time } \\
\text { (min)) }\end{array}$ & $75,3 \pm 0,68 * *$ & $71,34 \pm 0,75^{*}$ & $67,2 \pm 0,6$ \\
\hline Excretory function (cotton weight (mlg)) & $48,6 \pm 0,13^{* *}$ & $46,32 \pm 0,11 *$ & $41,2 \pm 0,08$ \\
\hline
\end{tabular}

The table shows that in patients with EOM, a slight change in the studied functions was revealed. On the contrary, in patients with EOM and $\mathrm{AR}$, there was a decrease in the protective function of the nasal mucosa and a shift in the $\mathrm{pH}$ of the nasal secretion to the alkaline side.

From the results of previous studies described in the literature, it is known that the $\mathrm{pH}$ shift of the nasal secretion to the alkaline side is one of the signs of serous inflammation occurring in the mucous membrane, including AR. Given the results of this study, we hypothesized the presence of a similar lesion of the nasal mucosa in patients with EOM and AR. It is more difficult to determine the possible causes of impaired protective function, based only on the results of this study. This is due, first of all, to the peculiarities of the structure of the mucociliary apparatus. In addition, there are many conditions that ensure the coordinated activity of all its structures and a significant number of external and internal factors that can cause their morphological and functional changes.

Comparing the results of this and previous studies, we believe that the most likely cause of the violation of the protective function of the nasal mucosa in patients with EOM and AR is serous inflammation, manifested by a number of structural and functional changes. Structural changes are characterized by de-epithelialization of the mucous membrane in the acute phase, an increase in the number of goblet cells, a change in the viscosity of the medium, and a change in the pH of the medium. Each of the described processes ultimately leads to a slowdown in mucociliary transport, which occurs in the patients with EOM and AR we examined.

\section{CONCLUSION}

Thus, the curvature of the nasal septum and other features of the architectonics of the intranasal structures in patients with EOM to a lesser extent than in patients with EOM and AR affect the development of the disease. The results of the study of the functions of the nose suggest the presence of serous inflammation of the nasal mucosa in patients with EOM and AR.

In addition, the results of a comparative analysis of the history and rhinoscopy data clearly demonstrate the dependence of relapses of exudative otitis media on the state of intranasal structures in patients of group 1 and the absence of such in patients of group 2. Therefore, we can assume that pathological changes in the intranasal structures are one of the main causes of the 


\section{The State of the Nasal Cavity of Patients with Exudative Otitis Media}

development of EOM. And we regard this factor only as an accompanying one. In further studies, it is required to determine the cause of the pronounced swelling of the nasal mucosa, identified in the majority of patients with EOM, and to assess its significance in the development of the disease.

\section{REFERENCES}

1) Mashkova T. A. et al. Leukotriene receptor blocker in the complex treatment of exudative otitis media in patients with allergic rhinitis // Modern problems of science and education. - 2021. - no. 3. - S. 124-124.

2) Savenko I. V., Boboshko M. Yu. Exudative otitis media: the main causes of development in childhood. Part I // Russian Bulletin of Perinatology and Pediatrics. - 2021. - T. 66. - No. 4. - S. 32-38.

3) Zolotova T. V., Manukyan A. G. Immune component in the pathogenesis of exudative otitis media in the pediatric age group // Russian otorhinolaryngology. - 2016. - no. 3 (82). - S. 174-175.

4) Sebekina O. V., Nenasheva N. M. The use of a new antihistamine drug in the treatment of allergic rhinitis // Consilium Medicum. - 2018. - T. 20. - No. 3.

5) Savenko I. V., Boboshko M. Yu. Exudative otitis media in children: the role of prematurity and concomitant pathology in its formation. Part II // Russian Bulletin of Perinatology and Pediatrics. - 2021. - T. 66. - No. 5. - S. 21-27.

6) Polunin M. M., Chernova O. V. Treatment of exudative otitis media in young children, taking into account the anatomical features of the auditory tube // Bulletin of Otorhinolaryngology. - 2020. - T. 85. - No. 1. - S. 10-13.

7) Burova O. V. et al. Balloon dilatation of the cartilaginous part of the auditory tube in children with recurrent exudative otitis media // Bulletin of Otorhinolaryngology. - 2016. - T. 81. - No. 2. - S. 59-60.

8) Narzullaev N. U., Suleimanov S. F. Analysis of the incidence of exudative otitis media in HIV-infected children // Modern trends in science, practice and education in pediatrics. - 2018. - S. 133-137.

9) Filanovich T. L. The relationship of exudative otitis media with the pathology of the nose, paranasal sinuses and nasopharynx. - 2018.

10) Zheltov A. Ya. et al. Etiopathogenetic substantiation of surgical treatment of nasopharyngeal diseases in children suffering from exudative otitis media. - 2019.

11) Roditi R. E., Shin J. J. The influence of age on the relationship between allergic rhinitis and otitis media //Current allergy and asthma reports. - 2018. - T. 18. - №. 12. - C. 1-9.

12) Sharifian M. R. et al. Correlation between allergic rhinitis and otitis media with effusion //Iranian journal of otorhinolaryngology. - 2019. - T. 31. - №. 105. - C. 209.

13) Nemati S. et al. Allergic rhinitis in adults with chronic suppurative otitis media //Iranian journal of otorhinolaryngology. 2015. - T. 27. - №. 81. - C. 261.

14) Altıntaş M., Muluk N. B., Peng K. A. What Is the Significance of Rhinitis in Otitis Media with Effusion? //Challenges in Rhinology. - Springer, Cham, 2021. - C. 159-168.

15) Cho C. G. et al. Effects of allergic rhinitis on the progression and recovery of acute otitis media in a mouse model //International Journal of Pediatric Otorhinolaryngology. - 2021. - T. 140. - C. 110497.

16) Hardani Sr A. K. et al. Risk Factors for Otitis Media in Children Referred to Abuzar Hospital in Ahvaz: A Case-Control Study //Cureus. - 2020. - T. 12. - №. 8.

17) Pau B. C., Ng D. K. Prevalence of otitis media with effusion in children with allergic rhinitis, a cross sectional study //International journal of pediatric otorhinolaryngology. - 2016. - T. 84. - C. 156-160.

18) Lee J. H. et al. A Case Report of Atopic Dermatitis with Otitis Media with Effusion and Chronic Rhinitis //The Journal of Korean Medicine. - 2021. - T. 42. - №. 1. - C. 110-118.

19) Sebekina O. V., Nenasheva N. M. The efficacy of a new antihistamine in the treatment of allergic rhinitis //Consilium Medicum. - 2018. - T. 20. - №. 3. - C. 67-72.

20) Zernotti M. E. et al. Otitis media with effusion and atopy: is there a causal relationship? //World Allergy Organization Journal. - 2017. - T. 10. - №. 1. - C. 1-9.

21) Khrykova A. G. The use of laser therapy in children with allergic rhinitis complicated by exudative otitis media //Russian Journal of Physiotherapy, Balneology and Rehabilitation. - 2018. - T. 17. - №. 5. - C. 258-262. 